\title{
Mild Encephalitis/Encephalopathy with Reversible Splenial Lesion Type II with New Spectrum
}

\author{
Nishant Nayyar ${ }^{1} \quad$ Lokesh Rana $^{1} \quad$ Dinesh Sood $^{1} \quad$ Indrajeet Singh Chauhan ${ }^{1} \quad$ Sudhir Yadav ${ }^{1}$ \\ 1Department of Radiodiagnosis, Dr. Rajendra Prasad Govt Medical \\ Address for correspondence Nishant Nayyar, MBBS, MD, \\ College, Tanda, Kangra, Himachal Pradesh, India \\ Department of Radiodiagnosis, Dr. Rajendra Prasad Govt Medical \\ College, Tanda 176001, Kangra, Himachal Pradesh, India \\ (e-mail: nishant23rpgmc@gmail.com).
}

Ann Natl Acad Med Sci (India) 2021;57:112-114.

\section{Introduction}

Mild encephalitis/encephalopathy with reversible splenial lesion is an acute encephalopathy with characteristic radiological findings. ${ }^{1}$ The typical form of mild encephalitis/encephalopathy with a reversible splenial lesion called Middle Eastern respiratory syndrome (MERS) type I is characterized by an isolated reversible lesion in the splenium of corpus callosum, while MERS type II refers to additional lesions that can occur in the rest of the corpus callosum and supratentorial white matter areas., ${ }^{2,3}$ Children and young adults are mainly affected, with favorable outcome leading to complete or nearly complete neurological and radiological resolution within days or weeks. ${ }^{4}$

\section{Case Report}

A 10-year-old male child presented in our hospital with abnormal body movements in the form of tonic clonic movements of both upper and lower limbs for the past 1 hour, associated with two episodes of vomiting and respiratory difficulty. Abnormal body movement episode was associated with uprolling of eyeballs and frothing from mouth.
No history of fever, upper respiratory tract infection, and trauma was present. Family history was not relevant. Development history was normal, and patient had been immunized to date. There was no history of recent travel and no past history of hospitalization. On admission, patient was afebrile, with pulse $78 / \mathrm{min}$, blood pressure $102 / 60 \mathrm{~mm}$ of $\mathrm{Hg}$, and $\mathrm{SPO}_{2} 86 \%$. On further physical examination, pupils were dilated and not reacting to light, his Glasgow coma scale (GCS) was E1VtM1 (no eye opening, intubated, no motor response) with no focal neurological signs or neck stiffness. Other systemic examinations were normal. Laboratory investigations revealed mildly elevated C-reactive protein (CRP) level ( $6.3 \mathrm{mg} / \mathrm{dl})$ and erythrocyte sedimentation rate (ESR) of $22 \mathrm{~mm} / \mathrm{hr}$ with normal leukocyte count $\left(9120 / \mathrm{mm}^{3}\right)$. Urine routine and microscopic examinations were normal with no growth of microorganisms on culture sensitivity. Serum electrolytes were normal during the course of treatment. Possibility of status epilepticus with respiratory failure, leading to hypoxic ischemic encephalopathy (HIE), was kept in mind. Management was started with mechanical ventilation, antibiotics, and loading doses of phenytoin. Lumbar puncture was done, which showed normal biochemical and published online July 28, 2021
DOI https://doi.org/

$10.1055 / \mathrm{s}-0041-1733302$ ISSN $0379-038 \mathrm{X}$
(C) 2021. National Academy of Medical Sciences (India).

This is an open access article published by Thieme under the terms of the Creative Commons Attribution-NonDerivative-NonCommercial-License, permitting copying and reproduction so long as the original work is given appropriate credit. Contents may not be used for commercial purposes, or adapted, remixed, transformed or built upon. (https://creativecommons.org/licenses/by-nc-nd/4.0/).

Thieme Medical and Scientific Publishers Pvt. Ltd. A-12, 2nd Floor, Sector 2, Noida-201301 UP, India 

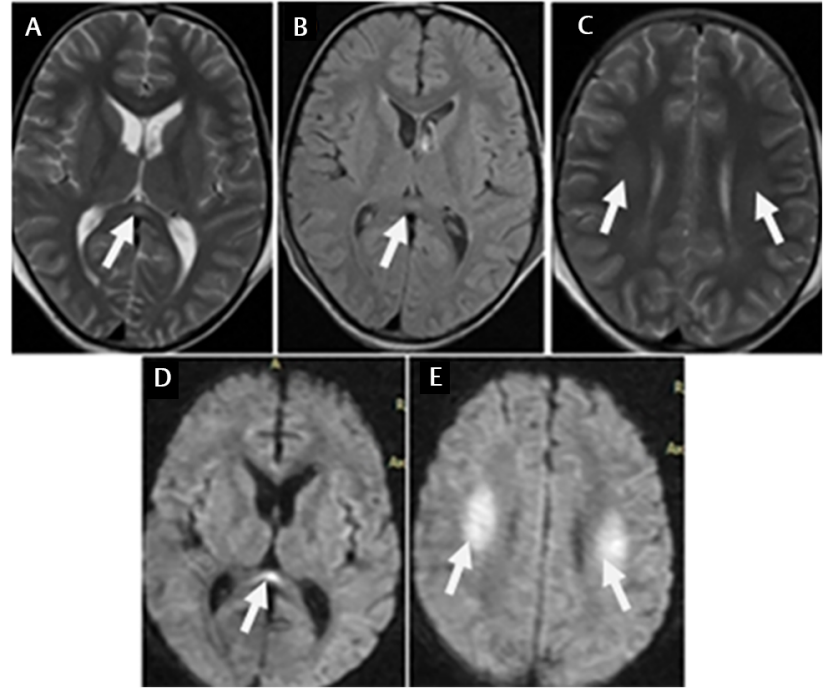

Fig. 1 Axial T2-weighted (A, C) and fluid-attenuated inversion recovery (FLAIR) (B) MRI shows subtle hyperintense area in the splenium of corpus callosum (arrow) and centrum semiovale (arrows). Diffusion-weighted imaging (DWI) (D, E) shows restriction in the corresponding regions, that is, splenium of corpus callosum and bilateral centrum semiovale.
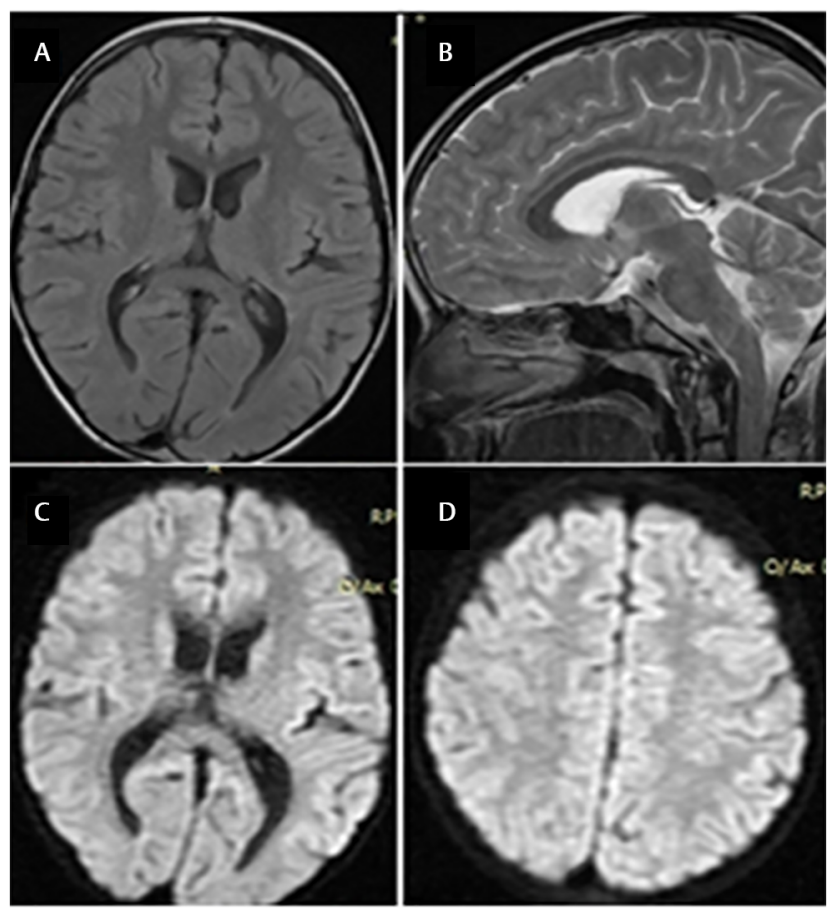

Fig. 2 Axial fluid-attenuated inversion recovery (FLAIR) (A), sagittal T2 and axial diffusion-weighted imaging (DWI) (C, D) shows resolution of the lesion, seen in the splenium of corpus callosum and centrum semiovale on follow-up after 2 months.

microbiological analyses. Patient underwent MRI on day 8 of admission. MRI T2-weighted (T2W) and fluid attenuated inversion recovery (FLAIR) images showed hyperintensity in the splenium of corpus callosum with bilaterally symmetrical hyperintensity in centrum semiovale ( - Fig. 1A-C). Diffusion-weighted Imaging (DWI) showed restriction in the

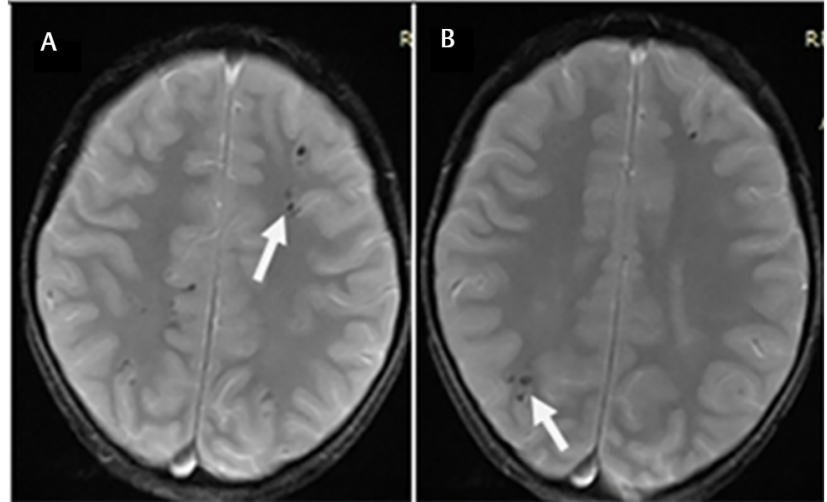

Fig. 3 Gradient echo (GRE) imaging (A, B) shows multiple punctate blooming foci in bilateral cerebral hemispheres on follow-up scan after 2 months, suggestive of microhemorrhages.

involved regions ( - Fig. 1D-E) without postcontrast enhancement or blooming on gradient echo (GRE) imaging, suggestive of type II MERS lesions. He was then empirically treated for encephalitis and improved after 40 days. A follow-up MRI scan 2 months later showed mostly resolved splenial and centrum semiovale lesions (- Fig. 2A-D); however, multiple punctate blooming foci were seen in white matter of bilateral cerebral hemispheres on GRE imaging, suggesting microhemorrhages ( - Fig. 3A-B).

\section{Discussion}

MERS is a clinicoradiological entity characterized by mild encephalopathy with typical radiological findings. ${ }^{1}$ The typical form of mild encephalitis with a reversible splenial lesion, also called MERS type I, is characterized by an isolated reversible lesion in the splenium of corpus callosum, while MERS type II refers to additional lesions that can occur in the rest of the corpus callosum and supratentorial white matter areas. ${ }^{2,3}$ The exact pathophysiology of this syndrome remains unknown; however, bacterial or viral infections remain the most common cause of reversible splenial lesions. Other conditions such as seizures, antiepileptic drug therapy or withdrawal, malnutrition, high-altitude edema, eclampsia, and metabolic disturbances have also been suggested as causes. $^{4-6}$ Presenting neurologic symptoms of MERS generally include altered consciousness, seizures, drowsiness, headache, abnormal speech, visual hallucinations, and ataxia., ${ }^{3,5-7}$

In addition to MERS, various other congenital to acquired diseases can involve splenium of corpus callosum, including congenital anomalies, ischemic changes, toxic/metabolic diseases, tumors, and demyelinating disorders. Clinical presentation and signs of these conditions are variable and can be differentiated from MERS with the help of imaging. The most common differential diagnoses include acute disseminated encephalomyelitis (ADEM), multiple sclerosis (MS), posterior reversible encephalopathy syndrome (PRES), and diffuse axonal injury, extrapontine myelinolysis. ${ }^{3-7}$ ADEM is a postinfectious inflammatory demyelinating disorder which should be considered in any patient presenting with mild acute encephalitis/encephalopathy. ADEM however 
has monophasic course with clinical recovery over weeks to months. On MRI, it shows multiple bilateral asymmetrical subcortical white matter lesions, which are hyperintense on T2/FLAIR images and may show enhancement. ${ }^{3-5}$ MS also show white matter lesions but in typical locations, that is, periventricular, juxtacortical, infratentorial and in spinal cord with callososeptal interface involvement of corpus callosum. PRES is a hypertension-related condition which show T2/FLAIR hyperintensities involving the subcortical white matter of the parieto-occipital lobes with isointense signal on DWI. Diffuse axonal injury is a traumatic brain injury with typical involvement of gray-white matter junction, corpus callosum, and brainstem, depending on the severity. MRI is the investigation of choice in suspected patients, which show small regions of susceptibility artifacts in typical aforementioned locations on gradient sequences. Extrapontine myelinolysis is an osmotic demyelination syndrome, which occur secondary to rapid correction of hyponatremia. It can involve basal ganglia, ventrolateral thalami, internal capsular region, splenium, and subcortical white matter. ${ }^{6,7}$

Our patient presented with seizure and altered consciousness. MRI findings we reported are almost similar to those reported earlier. ${ }^{1,4-7}$ The lesions typically show hyperintensity on T2W and FLAIR images, isointensity to slight hypointensity on T1-weighted imaging, and show restricted diffusion on DWI without contrast enhancement. Our patient, although improved, showed slow recovery with follow-up MRI showing blooming punctate foci, suggesting microhemorrhages. This has not been reported earlier, thus adding a new spectrum to the already described MERS.

\section{Conclusion}

MERS is an acute encephalopathy with characteristic imaging findings. The typical radiological lesions are reversible with complete clinical recovery in days or weeks. Our patient, although clinically improved, showed slow recovery with a new imaging spectrum that has not been reported earlier.

\section{Note}

Consent was obtained from relatives of the patient for publication of this report and any accompanying images.

\section{Author Contributions}

The authors confirm that the article is not under consideration for publication elsewhere, each of them sufficiently participated for the work to be submitted, and the publication is approved by all of them.

\section{Conflict of Interest \\ None declared.}

\section{References}

1 Tada H, Takanashi J, Barkovich AJ, et al. Clinically mild encephalitis/encephalopathy with a reversible splenial lesion. Neurology 2004;63(10):1854-1858

2 Takanashi J, Barkovich AJ, Shiihara T, et al. Widening spectrum of a reversible splenial lesion with transiently reduced diffusion. Am J Neuroradiol 2006;27(4):836-838

3 Takanashi J, Imamura A, Hayakawa F, Terada H. Differences in the time course of splenial and white matter lesions in clinically mild encephalitis/encephalopathy with a reversible splenial lesion (MERS. J Neurol Sci 2010;292(1-2):24-27

4 Ueda N, Minami S, Akimoto M. Mycoplasma pneumoniae-associated mild encephalitis/encephalopathy with a reversible splenial lesion: report of two pediatric cases and a comprehensive literature review. BMC Infect Dis 2016;16(1):671

5 Fang Q Chen L, Chen Q, Lin Z, Yang F. Clinically mild encephalitis/encephalopathy with a reversible splenial lesion of corpus callosum in Chinese children. Brain Dev 2017;39(4):321-326

6 Notebaert A, Willems J, Coucke L. Van Coster R, Verhelst H. Expanding the spectrum of MERS type 2 lesions, a particular form of encephalitis. Pediatr Neurol 2013;48(2):135-138

7 Kashiwagi M, Tanabe T, Shimakawa S, et al. Clinico-radiological spectrum of reversible splenial lesions in children. Brain Dev 2014;36(4):330-336 